\title{
A New Robust Nonlinear Control Algorithm for the Regulation of Blood Glucose in Diabetic Patients
}

\author{
Mine M. Ozyetkin ${ }^{1, \S}$, Nitendra Nath ${ }^{2}$, Enver Tatlicioglu ${ }^{3}$, and Darren M. Dawson ${ }^{4}$
}

\begin{abstract}
Diabetes mellitus is a growing health problem worldwide. Especially, the patients with Type 1 diabetes need strict glycemic control because they have deficiency of insulin production. Today, the aim of the researchers is to develop a fully automated closed loop control system (i. $e$., artificial pancreas which is capable of continuous glucose sensing and regulating) that replicates the function of pancreatic beta cells. This equipment is supposed to be capable of maintaining normal blood glucose level on a desired level (4-6.2 $\mathrm{mmol} / \mathrm{L}$ ) and taking all factors affecting glucose concentration into account without inducing hypoglycemia and hyperglycemia.

In this paper, control of the Bergman minimal mathematical model to regulate blood glucose level is discussed. The control problem is complicated by the fact that the model is considered to be uncertain. Furthermore, only the glucose level is assumed to be available for control development and other states such as insulin concentration and plasma insulin concentration are assumed to be unavailable for measurement. A novel robust controller is proposed that guarantees practical tracking of a desired glucose concentration.
\end{abstract}

\section{INTRODUCTION}

$\mathrm{D}$ IABETES is one of the most important chronic diseases [1]. According to the World Health Organization more than 180 million people suffer from diabetes worldwide, and it is estimated that this number will double in the next 30 years [1]-[3]. It is also predicted that approximately $9 \%$ of all deaths worldwide are caused by diabetes [4]. Diabetes mellitus emerges when the insulin secretion, a hormone generated in beta cells which regulates glucose concentration, decreases due to destruction of the pancreatic beta cells (Type 1 diabetes or insulin dependent) or the insulin is secreted but the body does not respond effectively (Type 2 diabetes or non-insulin dependent) [1], [3], [5]-[9].

In Type 1 diabetes, the pancreas does not release insulin since pancreatic beta cells are destroyed. So the effect of hormone glucagon raising blood glucose level cannot be suppressed by insulin. Thus, the patients need exogenous insulin injection to regulate their glucose concentration to a normal level. There are two situations depending on glucose

$\S$ To whom all the correspondence should be addressed.

1. M. M. Ozyetkin is with the Department of Electrical and Electronics Engineering, İnönü University, Malatya, 44280 Turkey (munevver.ozyetkin@inonu.edu.tr).

2. N. Nath is with Takata-Electronics, Pontiac, MI, 48341, USA, (nnath@alumni.clemson.edu).

3. E. Tatlicioglu is with the Department of Electrical \& Electronics Engineering, Izmir Institute of Technology, Izmir, 35430 Turkey (envertatlicioglu@iyte.edu.tr).

4. D. M. Dawson are with the Department of Electrical \& Computer Engineering, Clemson University, Clemson, SC, 29634 USA (ddarren@clemson.edu). concentration, namely, hyperglycemia and hypoglycemia. Hyperglycemia occurs if the glucose concentration is much higher than normal level (higher than $8 \mathrm{mmol} / \mathrm{L}$ ) [8]. Hypoglycemia, on the other hand, occurs when the glucose concentration is lower than normal level (lower than $3 \mathrm{mmol} / \mathrm{L}$ ) [8]. Both, hyperglycemia and hypoglycemia, can affect the patient's health and life. Kidney failure, heart disease, blindness, and other complications can be seen due to hyperglycemia in long term [1], [3], [4], [10], [11], while hypoglycemia has more critical affects in short term such as unconsciousness and coma or even death [4], [6], [8]-[10].

Some glucose monitoring technologies such as selfmonitoring blood glucose devices (SMBG) and continuous glucose monitoring (CGM) have been developed for glycemic control. SMBG devices are available commercially and the patients can check their blood glucose level by using them [4]. However, SMBG devices require four or more diurnally insulin injections [9]. As this method is painful, inconvenient and unreliable, it does not provide permanent observation and regulation. Therefore, it is quite difficult to avoid a hypoglycemic attack by using SMBG devices [1] CGM devices can have invasive or non-invasive sensors and require conventional finger stick measurements for recalibration [4]. In a recent study [4], it was reported that CGM devices are more successful for higher blood glucose levels and they are not reliable in detecting hypoglycemia.

From the control point of view, there are two insulin delivery strategies: open-loop and closed-loop [4]. Almost all commercially available insulin pumps have open-loop control that deliver pre-determined insulin dosage. Thus, they do not regulate insulin dosage depending on blood glucose level [4]. Furthermore, most insulin pumps that have open-loop control require attention of the user to operate.

As mentioned earlier, available treatment methods for Type 1 diabetes are subcutaneous insulin injection and permanently insulin infusion [1], [4], [9], [12]. Today, both of these treatment methods are indispensable. On the other hand, these treatments have some shortcomings. For instance, in subcutaneous insulin injection treatment, the patient may need three or more insulin injections coinciding to general meal times diurnally [4], [9], [12]. It depends on glucose measurement by using finger stick to determine amount of insulin injected to the body [9], which is both painful and inconvenient [1], [4], [6], [9]. Unfortunately, when other complications such as stress, illness, exercise, food intake and other disturbances are taken into consideration, it is possible to say that pre-determined dosage of insulin may easily become unreliable. Moreover, the episodic measurements may lead to an increasing 
incidence of hypoglycemia [1]. In contrast to this intermitted measurement method requiring finger prick measurement of blood glucose level, continuous glucose monitoring and sensing systems are more reliable [4].

As for insulin pumps, the most common problems are clogging and dislocating of the catheter, precipitation of insulin and inflammations around the located region. And the fact that they do not include a feedback system, they provide unreliable and ineffective treatment for the patients.

The realization of a fully automated closed-loop device (i.e., an artificial pancreas) capable of maintaining normal blood glucose level daily without inducing hypoglycemia and hyperglycemia will significantly improve the quality of life for individuals with diabetes especially with Type 1 diabetes [9]. This device is supposed to have three primary parts: (i) an insulin pump, (ii) glucose sensor, and (iii) control algorithm to regulate the amount of insulin delivered according to the blood glucose concentration measurements [6], [9], [13].

Researchers have studied several aspects of an artificial pancreas for over 40 years. However, a fully automated closed-loop device is not realized commercially yet [7]. Kadish et al. introduced a servo-mechanism for blood glucose control in the 1960s [3], [4], [7], [14]. Clarc and Lyons developed first glucose sensor in 1962 [4]. First commercial product, called biostator, was introduced by Clemens in the 1970s [3], [4], [9], [13]. It had two catheters, one to measure the glucose level and another one to deliver the required insulin. But, it was not appropriate for daily consumer use since it was nearly as big as a refrigerator [4]. Albissier studied artificial endocrine pancreas and developed a programmable hand-held device for self-management [15], [16]. Parker et al. [7], [9], [10] and Bequette et al. [7], [8], [10], [12] contributed to the field by studying model predictive control (MPC) applications. Farmer et al. studied different mathematical models such as Bergman minimal model, Hovorka model and Sorensen model and different control types such as proportional- integral-derivative (PID), feed-forward, and proportional [17]. Several control techniques such as PID control [6], [9], [17], [18], fuzzy-PID control [19], proportional derivative (PD) control [6], MPC [8], [9], [10], pole placement [6], [8], [9], run-to-run control [20], [21], $\mathrm{H}_{\infty}$ control [6], [22], fuzzy logic control [5] etc. have been employed to regulate glucose concentration. In the PID control, the amount of delivered insulin can be much higher than needed insulin level due to the integral term; therefore, it can cause postprandial hypoglycemia [7], [18]. Thus, as a result of the lack of the integrator term, PD control gives better results than PID [7]. MPC control is better than PD control [7], however, in this control type, the model has to be updated at every stage to compensate for difference between measured output and predicted output [7], and it utilizes a linear control algorithm to regulate nonlinear systems [9] which may result in instability. Runto-run control utilizes previous runs to obtain the input for the next run and it must be arranged individually for each patient [21]. Also, in the literature, most of the papers focus on linearization techniques (i.e., [6], [8], [9], [10], [12], [14], [18], [19], [22]) which may not represent the nonlinear characteristics of the blood glucose concentration dynamics. Review of the relevant past literature highlights the fact that for a better and accurate regulation of blood glucose in diabetic patients, a control algorithm should be developed which does not require exact knowledge of the patient parameters (i.e., robust). Further, the algorithm should only utilize measurement of the blood glucose concentration for the regulation of blood glucose in diabetic patients.

Some part of the past research focused on developing mathematical models to represent the dynamics of the blood glucose concentration. Several mathematical models describing relationships between insulin and glucose concentrations have been developed over the past years [10]. Bergman developed a mathematical model called the Bergman minimal model consisting of three ordinary differential equations to describe dynamics of the system [10], [17], [22]. Furler et al. introduced an extended form of the minimal model by omitting pancreatic insulin release and adding insulin antibodies to the original Bergman model [9], [17], [24]. Fisher developed a modified form by using the minimal model where insulin secretion term was omitted and an insulin infusion term was added [10]. The Bergman minimal model, the Sorensen model, the Hovorka model, the automated insulin dosage advisor (AIDA) model, the diabetes insulin advisory system (DIAS) model, and the Karlsburg diabetes advisory system (KADIS) model are some of the models in the literature [25].

In this paper, a new robust nonlinear control algorithm based on the Bergman minimal model is developed to regulate blood glucose concentration. Different from the most of the past research [6], [11], [14], [18], [19], [22], in this study, the mathematical model of the blood glucose concentration is assumed to be uncertain. Furthermore, different from [17], for the design to be realistic, only the glucose level is assumed to be available for control development and other states (insulin concentration and plasma insulin concentration) are assumed to be unmeasurable. The mathematical model is manipulated to form a compact form that includes only the blood glucose concentration and its time derivatives and then the error system is developed. Since only the blood glucose concentration is available, high gain observer signals are introduced to compensate for the lack of state measurements. The results from [26] can then be utilized to guarantee semiglobal, uniform and ultimate boundedness of the error signals.

\section{SYSTEM MODEL}

When compared with the other relevant studies in the literature, main advantages of the Bergman minimal model are the number of parameters is minimum [19] and it describes the interaction between main components such as insulin and glucose concentrations without getting into biological complexity. Thus, in this paper, the Bergman minimal model is considered [17] 


$$
\begin{aligned}
& \dot{G}=-p_{1}\left(G-G_{B}\right)+P-\left(X-X_{B}\right)\left(G-G_{B}\right) \\
& \dot{X}=-p_{2}\left(X-X_{B}\right)+p_{3}\left(I-I_{B}\right) \\
& \dot{I}=-n I+u / V_{1}
\end{aligned}
$$

where $G(t) \in R$ is the plasma glucose concentration in $\mathrm{mmol} / \mathrm{L}$ (or $\mathrm{mg} / \mathrm{dL}$ ) (above basal value), $X(t) \in R$ is proportional to the insulin concentration in the remote compartment in $m U / L, I(t) \in R$ is the plasma insulin concentration in $m U / L$ (above basal value), $P(t) \in R$ is the external glucose disturbance such as meal or injected glucose in mmol/Lmin, and $u(t) \in R$ is injected insulin rate in $\mathrm{mU} / \mathrm{min}$. In (1)-(3), $p_{1}, p_{2}, p_{3}, n \in R$ are constant patient parameters, $V_{1} \in R$ is the constant volume of the distributed insulin, and $G_{B}, X_{B}, I_{B} \in R$ are constant basal values of $G(t), X(t)$, and $I(t)$, respectively.

\section{CONTROL Problem}

In this section, the system model in (1)-(3) is mathematically manipulated to obtain a form that would allow us to facilitate the development of a robust controller. The control objective is to track a desired glucose concentration, denoted by $G_{d}(t) \in R$, with only using the measurements of $G(t)$. The control problem is further complicated by the fact that the mathematical model in (1)(3) is assumed to be uncertain.

To facilitate the control development, an auxiliary signal, denoted by $M(G) \in R$, is defined as follows

$M=1 /\left(G-G_{B}\right)$

Remark 1: It should be noted that, in this paper, hyperglycemia is considered; thus, the denominator of $M($. is strictly positive.

After multiplying both sides of (1) with $M($.$) , the following$ expression is obtained

$M \dot{G}=-p_{1}+M P-X+X_{B}$

After taking the time derivative of (5) and then rearranging the resulting expression, the following expression can be obtained

$M \ddot{G}=-\dot{M} \dot{G}+\dot{M} P+M \dot{P}-\dot{X}$

Substituting for $\dot{X}(t)$ from (2) into (6) results in

$M \ddot{G}=-\dot{M} \dot{G}+\dot{M} P+M \dot{P}+p_{2}\left(X-X_{B}\right)-p_{3}\left(I-I_{B}\right)$.

After taking the time derivative of (7), the following expression is obtained

$$
M \dddot{G}=-2 \dot{M} \ddot{G}-\ddot{M} \dot{G}+\ddot{M} P+2 \dot{M} \dot{P}+M \ddot{P}+p_{2} \dot{X}-p_{3} \dot{I} .
$$

After substituting for $\dot{I}(t)$ from (3) into (8), the following expression can be obtained

$M \dddot{G}=-2 \dot{M} \ddot{G}-\ddot{M} \dot{G}+\ddot{M} P+2 \dot{M} \dot{P}+M \ddot{P}+p_{2} \dot{X}$

$+p_{3} n I-p_{3} u / V_{1}$

Note that, from (5), following expression can be obtained for $X(t)$

$X=-M \dot{G}-p_{1}+M P+X_{B}$

From (10), it can be seen that $X(t)$ can be written as a function of $G(t)$ and $\dot{G}(t)$. It should also be noted that, after taking the time derivative of (10), it is possible to write $\dot{X}(t)$ in terms of $G(t), \dot{G}(t)$, and $\ddot{G}(t)$. Similarly, from (2), following expression can be obtained for $I(t)$

$I=1 / p_{3}\left(\dot{X}+p_{2}\left(X-X_{B}\right)\right)+I_{B}$.

Thus, after utilizing (10) and its time derivative along with (11), it is possible to write $I(t)$ in terms of $G(t), \dot{G}(t)$, and $\ddot{G}(t)$.

After multiplying both sides of (9) with $V_{1} / p_{3}$, following expression can be obtained

$V_{1} M \dddot{G} / p_{3}=V_{1} / p_{3}(-2 \dot{M} \ddot{G}-\ddot{M} \dot{G}+\ddot{M} P+2 \dot{M} \dot{P}+M \ddot{P}$

$\left.+p_{2} \dot{X}+p_{3} n I\right)-u$

$=V_{1} / p_{3}[-2 \dot{M} \ddot{G}-\ddot{M} \dot{G}+\ddot{M} P+2 \dot{M} \dot{P}+M \ddot{P}$

$+p_{2}(-\dot{M} \dot{G}-M \ddot{G}+\dot{M} P+M \dot{P})+n(-\dot{M} \dot{G}-M \ddot{G}+\dot{M} P+$

$\left.\left.M \dot{P}-p_{2} M \dot{G}-p_{1} p_{2}+p_{2} M P+p_{3} I_{B}\right)\right]-u$.

After utilizing the facts that $I(t)$ and $\dot{X}(t)$ can be written in terms of $G(t), \dot{G}(t)$ and $\ddot{G}(t)$, the following expression can be obtained

$\bar{M} \dddot{G}=f(G, \dot{G}, \ddot{G}, P, \dot{P}, \ddot{P})-u$

where $\bar{M}(t), f(t) \in \mathbb{R}$ are auxiliary signals defined as

$\bar{M}=V_{1} M / p_{3}$

$f=V_{1} / p_{3}[-2 \dot{M} \ddot{G}-\ddot{M} \dot{G}+\ddot{M} P+2 \dot{M} \dot{P}+M \ddot{P}$

$+p_{2}(-\dot{M} \dot{G}-M \ddot{G}+\dot{M} P+M \dot{P})+n(-\dot{M} \dot{G}-M \ddot{G}+\dot{M} P+$

$\left.\left.M \dot{P}-p_{2} M \dot{G}-p_{1} p_{2}+p_{2} M P+p_{3} I_{B}\right)\right]$.

It should be noted that, since only the plasma glucose concentration $G(t)$ is assumed to be measurable and the mathematical model in (1)-(3) is assumed to be uncertain, it is clear that $f(t)$ is not available for control design. 


\section{CONTROl DEVELOPMENT}

In this section, a robust controller will be designed to regulate the glucose level.

To quantify the control objective, a tracking error signal, denoted by $e_{1}(t) \in \mathbb{R}$, is defined as follows

$e_{1}=G_{d}-G$

where $G_{d}(t) \in \mathbb{R}$ is the desired glucose concentration and it is designed such its time derivatives up to fourth order are bounded functions of time.

To further facilitate the control development, auxiliary error signals, denoted by $e_{2}(t), e_{3}(t), r(t) \in \mathbb{R}$, are defined as

$e_{2}=\dot{e_{1}}+e_{1}$

$e_{3}=\dot{e_{2}}+e_{2}+e_{1}$

$r=e_{3}+e_{2}$

Since only the blood glucose concentration is assumed to be available, auxiliary error signals in (17), (18), and (19) are not available for control development. In the subsequent control development, high gain observers will be designed to compensate for the lack of these error signals. After taking the time derivative of $r(t)$, following expression can be obtained

$\dot{r}=\dddot{G}_{d}-\dddot{G}+\ddot{e}_{1}+2 \dot{e_{2}}+\dot{e_{1}}$

where (16)-(19) and their time derivatives were utilized. After multiplying both sides of (20) with $\bar{M}($.$) , the$ following expression can be obtained

$\bar{M} \dot{r}=\bar{M} \dddot{G}_{d}-f+u+\bar{M}\left(\ddot{e_{1}}+2 \dot{e_{2}}+\dot{e_{1}}\right)$

where (13) was utilized. The right-hand-side of the above expression can be rearranged to obtain the following form

$\bar{M} \dot{r}=N_{d}+\widetilde{N}-\frac{1}{2} \dot{\bar{M}} r-e_{3}+u$

where the auxiliary signals $\widetilde{N}(t), N_{d}\left(G_{d}, \dot{G_{d}}, \ddot{G}_{d}, \dddot{G}_{d}\right) \in \mathbb{R}$ are defined as

$\widetilde{N}=N-N_{d}$

$N_{d}=\left.N\right|_{G=G_{d}, \dot{G}=G_{d}, \ddot{G}=\ddot{G}}$

where the auxiliary signal $N(t) \in \mathbb{R}$ being defined as

$N=\bar{M} \dddot{G}_{d}-f+\bar{M}\left(\ddot{e}_{1}+2 \dot{e_{2}}+\dot{e_{1}}\right)+1 / 2 \dot{\bar{M}} r+e_{3}$.

Remark 2: Since $N_{d}($.$) is a function of G_{d}(t)$ and its time derivatives (which are designed as bounded functions of time), it is clear that $N_{d}(\cdot)$ and its time derivative are bounded functions.

Remark 3: The mean value theorem [27] can be utilized to prove that

$|\widetilde{N}().| \leq \rho(\|z\|)\|z\|$

where $\rho(.) \in \mathbb{R}$ is a non-decreasing positive function of its arguments and $z(t) \in \mathbb{R}^{4}$ is the combined error signal defined as

$z(t)=\left[e_{1} e_{2} e_{3} r\right]^{T}$.

As mentioned earlier, in this study, only the blood glucose concentration is considered to be available for control design; hence, the following high gain observers, denoted by $\widehat{e_{1}}(t), \widehat{e_{2}}(t), \hat{r}(t) \in \mathbb{R}$, are designed

$\dot{\hat{e_{1}}}=\widehat{e_{2}}-\widehat{e_{1}}+\alpha \alpha_{1} / \varepsilon\left(e_{1}-\widehat{e_{1}}\right)$

$\dot{\hat{\mathrm{e}_{2}}}=\hat{\mathrm{r}}-\widehat{\mathrm{e}_{2}}+{ }^{\alpha_{2}} / \varepsilon^{2}\left(\mathrm{e}_{1}-\widehat{\mathrm{e}_{1}}\right)$

$\dot{\hat{r}}=\alpha_{3} / \varepsilon^{3}\left(e_{1}-\widehat{e_{1}}\right)$

where $\alpha_{1}, \alpha_{2}, \alpha_{3}$, and $\varepsilon \in \mathbb{R}$ are positive observer gains. The control input $u(t)$ is designed as [26]

$u=-\operatorname{sat}\{(g+1) \hat{r}\}-\hat{f}$

where $\operatorname{sat}\{.\} \in \mathbb{R}$ is the saturation function, $g \in \mathbb{R}$ is a positive control gain, and $\hat{f}(t) \in \mathbb{R}$ is an estimated feedforward term.

Remark 4: It should be noted that the only restriction imposed on the term $\hat{f}(t)$ in (31) is that it should remain bounded throughout the closed-loop operation. The feedforward term is not directly specified here but in practice it can be implemented in several ways including neural network.

After substituting the above controller into the open-loop error system in (22), following closed-loop error system is obtained

$$
\bar{M} \dot{r}=N_{d}+\widetilde{N}-\frac{1}{2} \dot{\bar{M}} r-e_{3}-\operatorname{sat}\{(g+1) \hat{r}\}-\hat{f} .
$$

\section{STABILITY ANALYSIS}

Theorem 1: The output feedback controller in (31) yields a semi-globally, uniformly and ultimately bounded (SGUUB) tracking result in the sense that

$\left\|e_{i}(t)\right\| \leq \epsilon, i=1,2,3 \forall t \in\left[t_{0}+T, \infty\right)$

where $\epsilon, T \in \mathbb{R}$ are some positive constants. 
Proof: The proposed robust output feedback controller is a special case of the controller in [26], thus the stability analysis and the proof are omitted and reader is referred to [26] for a more detailed analysis.

\section{CONCLUSION}

A novel technique for regulation of blood glucose level for a diabetic patient with Type 1 diabetics was presented. A new robust control method was designed for a closed-loop system (i.e., artificial pancreas) to regulate the blood glucose concentration to a desired value. The control algorithm is designed despite an uncertain mathematical model and does not require exact knowledge of patient parameters. Only the blood glucose concentration was utilized in the control design (i.e., output feedback) and high gain observers were designed to compensate for the unavailable state signals. Future research will focus on simulation results by using numerical and/or practical examples.

\section{ACKNOWLEDGMENT}

The work of M. M. Ozyetkin was supported by TUBITAK (The Scientific and Technological Research Council of Turkey). Dr. Tatlicioglu's work is supported in part by Ministry of Science, Industry and Technology of Turkey (grant 1402.TGSD.2012).

\section{REFERENCES}

[1] K. Eskaf, O. Badawi, and T. Ritchings, "Predicting Blood Glucose Levels in Diabetics Using Feature Extraction and Artificial Neural Networks," in Proc. of Int. Conf. on Information and Communication Technologies: From Theory to Applications, Damascus, Syria, 2008, pp. 1-6.

[2] M. F. El Sharkawy, P. Georgiou, and C. Toumazou, "A silicon pancreatic islet for the treatment of diabetes," in Proc. of IEEE Int. Symp. on Circuits and Systems, Paris, France, 2010, pp.3136-3138.

[3] O. Vega-Hernandez, D. U. Campos-Delgado, and D. R. EspinozaTrejo, "Increasing security in an artificial pancreas: diagnosis of actuator faults," in Proc. of Pan-American Health Care Exchanges Conference, Mexico City, Mexico, 2009, pp. 137-142.

[4] V. R. Kondepati and H. M. Heise, "Recent progress in analytical instrumentation for glycemic control in diabetic and critically ill patients," Anal Bional Chem, 388, pp. 545-563, 2007.

[5] N. Jayaraj, C. M. Cherian, and S. G. Vaidyanathan, "Intelligent insulin infuser," in Proc. of the Third European Symp. on Computer Modeling and Simulation, Athens, Greece, 2009, pp. 74-78.

[6] S. Yasini, A. Karimpour, M. B. Naghibi-Sistani, and S. Ghareh,"An automatic insulin infusion system based on H-infinity control technique," 2008 Cairo International Biomedical Engineering Conference, Cairo, Egypt, 2008, pp. 1-5.

[7] B. W. Bequette,"A critical assessment of algorithms and challenges in the development of a closed-loop artificial pancreas,"Diabetes Technology \& Therapeutics, vol. 7, no.1, pp. 28-47, 2005.

[8] S. M. Lynch and B. W. Bequette,"Estimation-based model predictive control of blood glucose in type I diabetics: A simulation study," Proceedings of the IEEE 27th Annual Northeast Bioengineering Conference, Storrs, CT, USA, 2001, pp. 79-80.

[9] R. S. Parker, F. J. Doyle and N. A. Peppas, "The intravenous route to blood glucose control, a review of control algorithms for noninvasive monitoring and regulation in type I diabetic patients,"IEEE Engineering in Medicine and Biology, vol. 20, no. 1, pp. 65-73, 2001.

[10] S. M. Lynch and B. W. Bequette, "Model predictive control of blood glucose in type 1 diabetics using subcutaneous glucose measurements," Proceedings of the American Control Conference, Anchorage, AK, USA, 2002, pp. 4039-4043.
[11] J. Ahmed, B. A. Alvi and Z. A. Khan, "Blood glucose-insulin regulation and management system using MATLAB/SIMULINK," International Conference on Emerging Technologies IEEE-ICET, Rawalpindi, Pakistan, 2008, pp. 304-308.

[12] B. W. Bequette, "Optimal estimation of blood glucose," Bioengineering Conference, Proceedings of the IEEE $30^{\text {th }}$ Annual Northeast, Springfield, MA, USA, 2004, pp. 77-78.

[13] S. Shalitin and M. Philip, "Closing the Loop: Combining Insulin Pumps and Glucose Sensors in Children With Type 1 Diabetes Mellitus," Pediatric Diabetes, 7 (Suppl. 4), 45-49, pp. 45-49, 2006.

[14] B. Gopakumaran, H. M. Duman, D. P. Overholser, I. F. Federiuk, M. J. Quinn, M. D. Wood and W. K. Ward, "A novel insulin delivery algorithm in rats with type 1 diabetes: the fading memory proportional-derivative method," Artificial Organs, vol. 29, no. 8, pp. 599-607, 2005.

[15] A. M. Albisser, " Toward algorithms in diabetes self-management," Diabetes Technology \& Therapeutics, vol. 5, no.3,pp. 371-373, 2003.

[16] A. M. Albissier, "Devices for the control of diabetes mellitus," Proceedings of the IEEE, vol. 67, no. 9, pp.1308-1319, 1979.

[17] T. G. Farmer, T.F. Edgar and N. A. Peppas, "Effectiveness of intravenous infusion algorithms for glucose control in diabetic patients using different simulation models," Ind. Eng. Chem. Res., vol.48, pp. 4402-4414, 2009.

[18] G. Marchetti, M. Barolo, L. Jovanovic, H. Zisser and D. E. Seborg, "An improved PID switching control strategy for type 1 diabetes,"IEEE Transactions on Biomedical Engineering, vol. 55, no. 3, pp. 857-865, 2008.

[19] C. Li, and R. Hu,"Fuzzy-PID control for the regulation of blood glucose in diabetes,"Global Congress on Intelligent Systems, Xiamen, China,2009, pp. 170-174.

[20] H. Zisser, L. Jovanovic, F. Doyle, P. Ospina and C. Owens, "Run-torun control of meal-related insulin dosing," Diabetes Technology \& Therapeutics, vol. 7, no.1,pp. 48-57, 2005.

[21] C. Owens, H. Zisser, L. Jovanovic, B. Srinivasan, D. Bonvin and F. J. Doyle, "Run-to-run control of blood glucose concentrations for people with type 1 diabetes mellitus," IEEE Transactions on Biomedical Engineering, vol. 53, no. 6, pp. 996-1005, 2006.

[22] K.H.Kienitz, and T. Yoneyama, "A robust controller for insulin pumps based on $H$-infinity theory, "IEEE Transactions on Biomedical Engineering, vol. 40, no. 11, pp. 1133-1137, 1993.

[23] R. N. Bergman, Y. Z Ider, C. R Bowden and C. Cobelli, "Quantitative estimation of insulin sensitivity," AJP: Endocrinology and Metabolism, vol. 236, no. 6, pp. E667-E677, 1979.

[24] S. M. Furler, E. W. Kraegen, R. H. Smallwood and D. J. Chisolm, "Blood glucose control by intermittend loop closure in the basal mode: computer simulation studies with a diabetic model," Diabetes Care, vol.8, pp.553-561, 1985 .

[25] G. M. Steil, B. Clark, S. Kanderian and K. Rebrin, "Modeling insulin action for development of a closed-loop artificial pancreas," Diabetes Technology \& Therapeutics, vol. 7, no.1, pp. 94-108, 2005.

[26] J. Chen,A. Behal, and D. M. Dawson, "Robust feedback control of uncertain MIMO nonlinear systems," IEEE Transactions on Automatic Control, vol.53, no. 2, pp. 591-596, 2008.

[27] H. K. Khalil, Nonlinear Systems, 3rd Edition, New York, NY, USA Prentice Hall, 2002. 